XXXIVth International Conference on High Energy Physics (ICHEP 2008), Philadelphia, Pennsylvania, USA, July 30-August 5, 2008.

\title{
Searches for hyperbolic extra dimensions at the LHC
}

\author{
Tommy Ohlsson \\ Department of Theoretical Physics, Royal Institute of Technology (KTH), 10691 Stockholm, Sweden \& \\ Royal Swedish Academy of Sciences (KVA), P.O. Box 50005, 10405 Stockholm, Sweden
}

\begin{abstract}
In this poster, we present a model of large extra dimensions where the internal space has the geometry of a hyperbolic disc. Compared with the ADD model, this model provides a more satisfactory solution to the hierarchy problem between the electroweak scale and the Planck scale, and it also avoids constraints from astrophysics. Since there is no known analytic form of the Kaluza-Klein spectrum for our choice of geometry, we obtain a spectrum based on a combination of approximations and numerical computations. We study the possible signatures of our model for hadron colliders, especially the LHC, where the most important processes are the production of a graviton together with a hadronic jet or a photon. We find that for the case of hadronic jet production, it is possible to obtain relatively strong signals, while for the case of photon production, this is much more difficult.
\end{abstract}

\section{INTRODUCTION AND THE HIERARCHY PROBLEM}

One of the main motivations for large extra dimensions is that they provide a solution to the so-called hierarchy problem between the electroweak scale $M_{\mathrm{ew}} \simeq 100 \mathrm{GeV}$ and the Planck scale $M_{\mathrm{Pl}} \equiv G_{N}^{-1 / 2} \simeq 10^{19} \mathrm{GeV}$. Theoretically, $M_{\text {ew }}$ is expected to obtain loop corrections of order $M_{\mathrm{Pl}} \gg M_{\mathrm{ew}}$. Therefore, a miraculous cancellation is needed to keep $M_{\text {ew }}$ at the order of $100 \mathrm{GeV}$. The so-called ADD (Arkani-Hamed, Dimopoulos, and Dvali) model [1] provides a very elegant solution to this problem. However, there is a problem related to this solution in the ADD model, since it turns out that it just becomes a reformulation, which means that the ADD model still suffers from the hierarchy problem. Nevertheless, in models with hyperbolic geometry [2], this problem can be remedied.

\section{THE HYPERBOLIC DISC MODEL}

The model that we consider is similar to the ADD model, with the only exception that the internal space is a two-dimensional hyperbolic disc, with constant negative curvature $v[3]$. The SM fields are assumed to be confined to a four-dimensional brane, while gravity alone probes the extra dimensions. See Fig. 1 for an illustration.

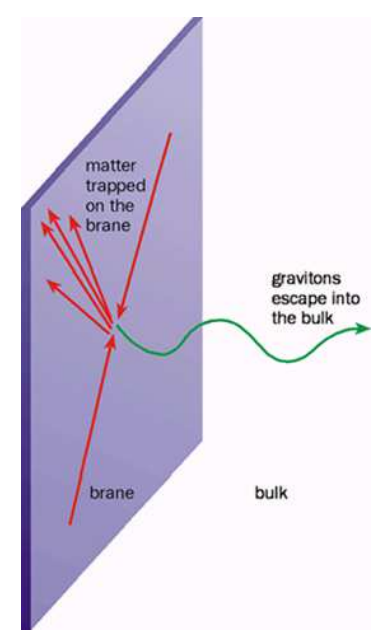

Figure 1: An illustration of the brane and the extra dimensions. The figure has been adopted with permission from [4].

In the ADD model, the Planck scale $M_{\mathrm{Pl}}$ is replaced by a new mass scale $M_{*}$. The two scales are related through 
XXXIVth International Conference on High Energy Physics (ICHEP 2008), Philadelphia, Pennsylvania, USA, July 30-August 5, 2008.

the equation $M_{\mathrm{Pl}}^{2}=V M_{*}^{4}$. If $V$ is large enough, then $M_{*}$ could be as low as $M_{\mathrm{ew}} \simeq 1 \mathrm{TeV}$, hence eliminating the hierarchy problem between $M_{\mathrm{Pl}}$ and $M_{\text {ew }}$. However, in the ADD model, the radius $L \simeq 10^{31 / d} M_{*}$, and thus, a new hierarchy problem is created, which means that the hierarchy problem is only reformulated as the question of why the radius of the internal space is so large compared to the electroweak scale

The area of a hyperbolic disc with radius $L$ is

$$
V=\frac{4 \pi}{v^{2}} \sinh ^{2}\left(\frac{v L}{2}\right) \sim \frac{\pi}{v^{2}} \exp (v L) .
$$

This has the result that, if the internal space is a hyperbolic disc, then it is possible to have $M_{\mathrm{Pl}} \simeq M_{\mathrm{ew}} \simeq L^{-1}$, i.e., no new large hierarchy is introduced, if also $v \simeq M_{*}$.

\section{COMPARISON WITH THE ADD MODEL}

Now, we perform a comparison between the ADD model and the hyperbolic disc model. First, there are the properties of the ADD model: one free parameter, i.e., $M_{*}$; the Kaluza-Klein $(\mathrm{KK})$ spectrum starts out at $m \approx 0$; for $d=2, M_{*}$ is constrained to $M_{*} \geq 50 \mathrm{TeV}$ from astrophysics; universal coupling of KK modes; physical results are independent of the position of the brane; and an exact solution for the KK spectrum. Second, there are the properties of the hyperbolic disc model: three free parameters, i.e., $M_{*}, v$, and $\tau_{b}$, where $\tau_{b}$ is the radial position of the brane in the internal space; the KK spectrum starts out at $m \approx v / 2>0$; weak restrictions on the parameter space; different KK modes have different couplings to SM fields; physical results depend on the position of the brane; and no exact solution for the KK spectrum, which means that approximations are needed. Thus, we observe that in the hyperbolic disc model we have more parameter freedom, a mass gap, and especially, the hierarchy problem can be solved. However, the results will depend on the position of the brane and there is no analytic solution for the KK spectrum.

\section{LHC PHENOMENOLOGY}

The KK modes of the graviton couple to all SM fields. We have considered possible production of KK gravitons at the LHC. Since each individual KK mode couples very weakly to SM fields, we need to study the production of any kinematically available KK mode. Because of the weak couplings, the graviton is not observed in detectors, and hence, we consider production of a graviton together with some observable particle. At the LHC, there are two interesting reactions: $p+p \rightarrow$ jet $+G$ and $p+p \rightarrow \gamma+G$. See Fig. 2 for the Feynman diagrams of the two reactions.
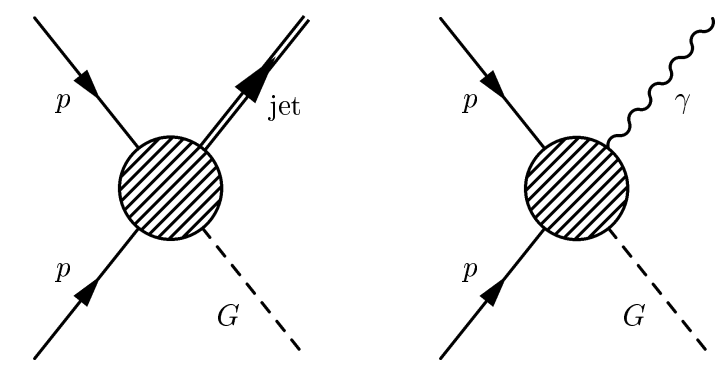

Figure 2: The Feynman diagrams for the reactions $p+p \rightarrow$ jet $+G$ (left) and $p+p \rightarrow \gamma+G$ (right).

\section{RESULTS}

For both of the reactions mentioned above, we have computed the differential cross sections with respect to the transverse momentum $p_{\mathrm{T}}$ of the outgoing jet/photon, and with respect to $\cos (\theta)$, where $\theta$ is the angle between the 
XXXIVth International Conference on High Energy Physics (ICHEP 2008), Philadelphia, Pennsylvania, USA, July 30-August 5, 2008.

beam and the jet/photon. As a reference, we also give the corresponding results for the ADD model, as well as the SM background, which mainly comes from the processes $p+p \rightarrow$ jet $/ \gamma+Z(\rightarrow \nu \bar{\nu})$.

In Fig. 3. the jet production results that could be observed at the LHC are shown as functions of $p_{\mathrm{T}}$ and $\cos (\theta)$, respectively, whereas in Fig. 4, the photon production results are shown as functions of $p_{\mathrm{T}}$ and $\cos (\theta)$, respectively.
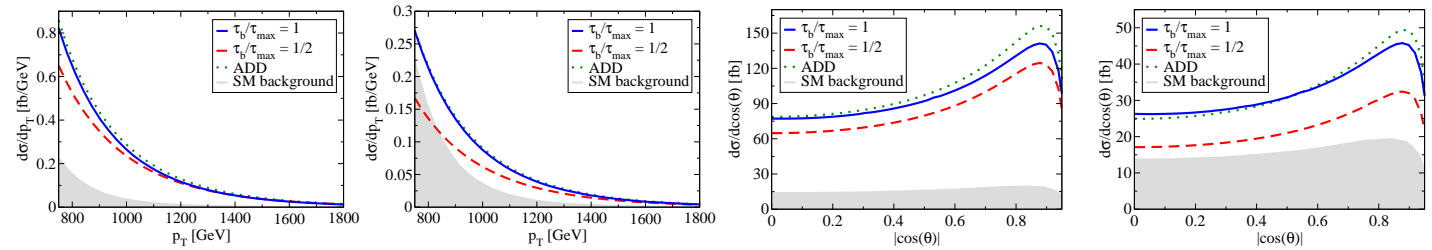

Figure 3: The differential cross section for graviton plus jet production with respect to $p_{\mathrm{T}}$ and $\cos (\theta)$, respectively. Left-left panel: $M_{*}=1.5 \mathrm{TeV}$. Left-right panel: $M_{*}=2 \mathrm{TeV}$. Right-left panel: $M_{*}=1.5 \mathrm{TeV}$. Right-right panel: $M_{*}=2 \mathrm{TeV}$.
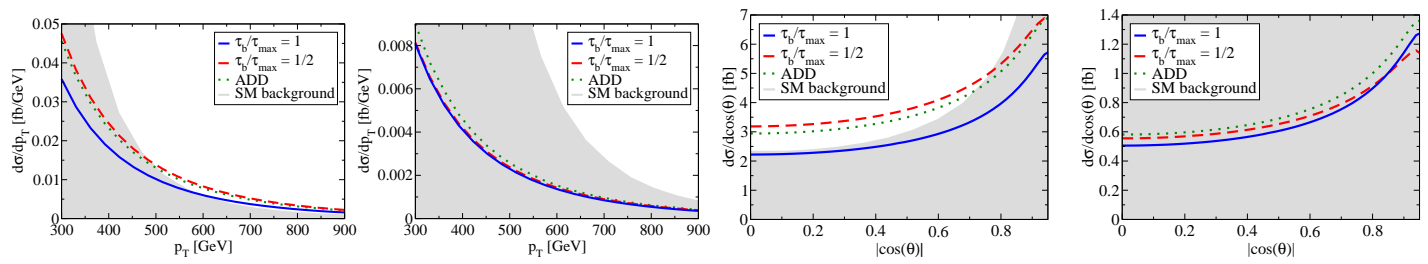

Figure 4: The differential cross section for graviton plus photon production with respect to $p_{\mathrm{T}}$ and $\cos (\theta)$, respectively. Left-left panel: $M_{*}=1 \mathrm{TeV}$. Left-right panel: $M_{*}=1.5 \mathrm{TeV}$. Right-left panel: $M_{*}=1 \mathrm{TeV}$. Right-right panel: $M_{*}=1.5 \mathrm{TeV}$.

\section{CONCLUSIONS}

In conclusion, we have found that i) the signals are similar to those of the ADD model, ii) the most promising signal comes from the jet channel, and finally, iii) it is more difficult to obtain an observable signal from the photon channel.

\section{Acknowledgments}

I would like to thank Henrik Melbéus for useful collaboration that led to the publication [3] upon which this poster is based. In addition, I would like to thank the organizers of ICHEP 2008 for the possibility to show the poster.

This work was supported by the Royal Swedish Academy of Sciences (KVA) and the Swedish Research Council (Vetenskapsrådet), Contract No. 621-2005-3588.

\section{References}

[1] N. Arkani-Hamed, S. Dimopoulos, and G.R. Dvali, "The Hierarchy Problem and New Dimensions at a Millimeter", Phys. Lett. B429, 263 (1998), hep-ph/9803315.

[2] N. Kaloper, J. March-Russell, G.D. Starkman, and M. Trodden, "Compact Hyperbolic Extra Dimensions: Branes, Kaluza-Klein Modes, and Cosmology", Phys. Rev. Lett. 85, 928 (2000), hep-ph/0002001.

[3] H. Melbéus and T. Ohlsson, "Searches for hyperbolic extra dimensions at the LHC", J. High Energy Phys. 08, 077 (2008), arXiv:0806.1841 [hep-ph].

[4] S. Abel and J. March-Russell, "The search for extra dimensions", Physics World, November, 38 (2000), http://physicsworld.com/cws/article/print/403/1/pw1311093 